\title{
Vinson über Iberisch und Baskisch.
}

Vinson hatte in der Revue de linguistique 1907 S. 1-23 (La langue ou les langues ibériennes) von neuem die iberisch-baskische Verwandtschaft bestritten; ich kritisierte diesen Aufsatz am Schlufs meiner Abhandlung "Die iberische Deklination" (SB. der Wiener Ak. Ph.-h. Kl. 157, 2), welche Ende Mai im Drucke vorlag; Vinson hat nun darauf, mit Ausdehnung auf meine ganze Abhandlung, in jener Zeitschrift erwidert, S. 209-237 (L'ibère et le basque; réponse à H. Schuchardt). Ich würde mir eine Replik ersparen, gäbe es nicht nach wie vor Leute welche meinen: da ein so guter Kenner des Baskischen wie Vinson in den Überbleibseln des Iberischen nichts Baskisches zu entdecken vermöge, so werde es wohl mit dieser Verwandtschaft auch in der Tat nichts sein. So muls ich denn die Fundamente tiefer legen. Wenn Vinson sagt (S. 2 I I f.): "Le travail de Sch. ne démontre point que je me sois trompé. Il fait des hypothèses différentes, voilà tout", so sage ich: nein, das ist nicht alles, auch unsere Methoden sind verschieden, und es kommt darauf an festzustellen ob die Bekenner der Gleichung Iliberri = Iliberri "Baskomanen" sind oder ob nicht vielmehr die Manie auf Seiten der Leugner $z u$ suchen ist. Ähnliche Vergleichungen wie die zwischen Iberisch und Baskisch werden aut so manchen andern Gebieten vorgenommen und über die zu befolgenden Grundsätze herrscht im grofsen ganzen keine Meinungsverschiedenheit. $\mathrm{Zu}$ einem positiven Ergebnis gelangt man dabei mit Hilfe verschiedener Elemente, die an sich sicher, wahrscheinlich oder möglich sind und durch ihre Zusammenfügung an Beweisfähigkeit gewinnen. Der Gegenpart muls diesem Verfahren folgen; er darf nicht wie es Vinson tut, das oder jenes, gleichsam als Stichprobe, aus dem Bau herausreifsen und unter Benutzung des Umstandes dals die sprachgeschichtliche Gewilsheit nicht an die mathematische heranreicht, auch das Bestbegründete mit dem Worte zu entwerten versuchen: "aber erwiesen ist es doch nicht". Und dann seinerseits Fragen und Einfälle in bunter Menge ausstreuen und jedem Einwand mit dem Worte begegnen: naber möglich ist es doch". Manchmal freilich wendet er sich ohne weiteres zum Rückzug; so spricht er von gewissen, für mich übrigens gar nicht auffälligen Umständen der iberischen Inschriften welche 
auf den Gedanken bringen könnten „que le déchiffrement n'a pas dit son dernier mot [wer behauptet denn das?] et qu'il y avait peut-être là une écriture mystérieuse, ancienne, un langage de convention", und setzt sofort hinzu: "Je ne le crois pas pourtant" (S. 2 I I). Inschriftendeutungen welche, seien sie auch verfehlt, doch auf einer bestimmten Grundlage ruhen, nennt er "fantaisistes", seine eigenen ganz aus der Luft gegriffenen "hypothétiques“; „mais je n'y tiens en aucune façon" (S. 230). Kurz, Vinson fegt wie ein Sturmwind über die Oberfläche dahin, wirbelt alles Bewegliche auf, hüllt alles Feste in eine Staubwolke. Ich erkläre mir das damit dafs er mehr aus einem Gefühl der Verpflichtung als aus innerer Neigung sich dieser Angelegenheit widmet; sonst würde er wohl auch mit derselben Sorgfalt vorgehen wie bei seinen bibliographischen Arbeiten und nicht solchen Massen von Druckfehlern den Eingang verstatten. Diese sind gerade hier sehr störend; in derartigen Erörterungen müssen wirklich „die Tüpfelchen auf die $i$ gesetzt werden".

Vinsons Aufsatz enthält eine längere Einlage in der er seine Ansichten über den alten Stand des Baskischen vorlegt. Zum Teil handelt es sich dabei um Bekanntes und Anerkanntes; eine Beziehung auf die „iberische Frage" oder auf meine Abhandlung nehme ich hier überhaupt nicht wahr, ebensowenig hat er, wie eine "Antwort" an mich wohl erwarten liefs, bei passenden Gelegenheiten meine andern Arbeiten berücksichtigt, mit Ausnahme eines Falles. 1

1 (S. 216 Anm.) V. vermutet dals das $-r$. welches sich in gewissen Formen des transitiven Hilfsverbs mit Zielpronomen findet, dem faktitiven -ra. entspreche, dafs also z. B. eman daraut "il me l'a donné" eigentlich soviel wäre wie: "il le fait avoir à moi donné". "Aber wenn hier die besondere Bedeutung des Hauptverbs eine kleine Stütze gewährt, so versagen andere Fälle eine solche; z. B. hartu daraut "il me l'a pris" läfst sich kaum fassen als: „il le fait avoir à moi pris". Ausserdem stünden mit einem so gedeuteten daraut im Widerspruch andere Formen desselben Verbs mit Zielpronomen (wie diczadan "qu'il me l'ait") ebenso wie gleichwertige Formen anderer Mundarten (wie bizk. deust "il me l'a"). Ich kenne keine ernstlichen Bedenken die gegen meine Herleitung des - $r$ - aus dem - $d$ - des Verbs - $(d) u$ - (Bask. Stud. I, 45) erhoben worden wären. Jenes -ra. möchte V. auch in dem transitiven ezaerkennen, dessen Identität mit dem intransitiven iza. mir aufser $Z$ weifel steht (a. a. O. I, $20 \mathrm{ff}$ ); die Verwandlung des stimmhaften $-r$ - in das stimmlose $\cdot z$. wäre ohne Beispiel, und auf ezarri "setzen" neben jarri "sich setzen" darf er sich nicht berufen. - (S. $217 \mathrm{ff}$.) Die Theorie von dem passiven Charakter des bask. Transitivs, welche von Stempf nicht allein und nicht zuerst vertreten worden ist, stellt V. eine andere, ${ }_{n}$ bien meilleure" gegenüber. Sie ist aber in so nebelhaften Umrissen gezeichnet dafs man sie nicht näher ins Auge fassen kann, und das wird auch wohl ebensowenig verlangt wie "on ne me demandera pas, je l'espère, de justifier ici les restitutions que je propose". Nur die bestimmt geäufserte Möglickkeit dals bask. nakus "er sieht mich" einst dem madj. látok "ich sehe" (ohne Objekt) entsprochen" habe, weise ich nachdrücklichst zurück. Und ebensowenig vermag ich mir vorzustellen wie dauntza „sie liegen“ ursprünglich ein „imparfait indéterminé" gewesen wäre; das $n$ stammt allerdings, wie ich ja an der von $\mathrm{V}$. angeführten Stelle gesagt habe, aus dem Imperfekt, aber zufolge einer Analogie. Das au für a möchte 
Die Einwürfe Vinsons gegen meine „Iber. Dekl." erledige ich unter einzelnen Schlagwörtern.

Iberisch, Aquitanisch, Baskisch. Hier liegt einer der Hauptpunkte der "Frage“. Für mich ist das Aquitanische, von dem uns ein paar hundert Personen- und Götternamen eine ziemlich deutliche Vorstellung geben, diejenige iberische Mundart auf welche das Baskische in gerader Linie zurückgeht. In ausführlicher und sorgfältiger Weise war dieses Aquitanisch behandelt worden von A. Luchaire in seinen Études sur les idiomes pyrénéens de la région française 1879 . Man hätte erwarten sollen dals niemand sich eifriger und gründlicher, sei es ablehnend, sei es beistimmend, mit dem Buche beschäftigen würde als der Herausgeber der Revue de linguistique. Aber er zeigte es hier (noch 1879; S. 339 f.) mit nur wenigen Worten an, indem er hinzusetzte: „je me propose de revenir sur cet intéressant ouvrage. J'aurais notamment à présenter de nombreuses observations sur toute la partie du volume qui est relative à la langue basque, à son extension probable ancienne,

V. als "renforcement vocalique a ansehen. Aber was ist damit gemeint? wodurch wird eine solche Verstärkung hervorgerufen? Ich fürchte dafs hier Guṇa und Vrrddhi spuken. Das au von erauntzi (-tsi?) soll ebenso aus dem $a$ von erasi entstanden sein; aber die Sache verhält sich umgekehrt (vgl. Bask. Stud. I, 42 f.). - (S. $220 \mathrm{Anm}$.) V. bemerkt dafs gewisse intransitive Verben im Bask. der transitiven Konjugation folgen, z. B. iluzkiak [welcher Md. gehört diese Form an? einer hochnav.?] argitzen du "die Sonne leuchtet", eig. nleuchtet es". Davon seien solche Fälle zu trennen wie arreit niri "folge mir"; aber das hat ja gar nichts besonderes an sich, warum tührt er nicht vielmehr darrayot (mundartlich neben narrayo Bask. Stud. I, 4I) an, das eigentlich ist: "ich folge es ihm", also vollständig zum ersten Beispiel pafst (ich habe solche Verben unpersönlich-transitive genannt; jenes ist ziellos, dieses zielend)? - (S. 213) Eine Gruppe von Erscheinungen die ich in meinem "Bask. u. Rom." zu ordnen und zu ergründen mich ernstlich bemüht habe, in der Hoffnung dals andere die Arbeit fortsetzen würden, streift hier V. mit ein paar Worten, nämlich den Schwund und Zutritt anlautender Konsonanten im Bask. Ich bemerke dazu dafs in garrathoin neben arrathoin "Ratte" und in katabute neben atabute "Sarg" südfranz. garri "Ratte" und catafau "Katafalk" hereinspielen, difs gaztigar neben aztigar, nicht sowohl "Linde" als (nach Lacoizqueta und Azkue) "Ahorn", das Ursprünglichere ist (von süd-


Ahorn" \} ihar, igar "trocken", "steif"; vgl. südfranz. asedur "Ahorn" \} acer durumj und dals das aus dem folgenden $w$ entsprungene $g$ von span. guada [vielmehr guadi-, guad.] \} arab. wädi hier auch nicht vergleichsweise heranzuziehen ist. - (S. 222) Gelegentlich der Abneigung des Baskischen gegen anlautende Tenues erinnert V. daran "qu'un allemand parlant français durcira ou adoucira une explosive là où, dans sa propre langue, il prononcerait une douce ou une dure ${ }^{4}$. Sollte V. in der Tat nichts davon wissen dafs die grölsere Hälfte der Deutschen keine Media kennt, und sollte er das schlechte Gehör der französischen Romanschriftsteller teilen, welche z. B. détestable aus deutschem Munde als tédesdaple hören? Unmöglich wäre es nicht, bringt er doch in demselben Heft seiner Revue unter dem Titel "Les beautés de l'Allemand" - offenbar um seine Landsleute zum Studium des Deutschen anzueifern - eine mit Druckfehlern gewürzte, sonst aber ganz ungesalzene Geschichte die in der Zusammensetzung gipfelt: Hottentotenstrottertrotelmutterattentaterlattengitterwetterkotterbeutelratte. 
etc., etc. " Ich wüIste nicht dafs er diese Absicht je ausgeführt hätte, und auch die von mir ihm gebotene Gelegenheit auf das Aquitanische zurückzukommen, hat er sich nicht zunutze gemacht. Er sagt (S. 235): „Pour prouver que le basque a été parlé sur un territoire plus étendu qu'aujourd'hui, on a cité des noms à apparence basque qui figurent dans des exvoto et des inscriptions de toute la région pyrénéenne; mais cela peut simplement être le fait de voyageurs, comme la pierre de Cagliari et l'urne de Sicile sont des faits accidentels." Er fügt kein Wort hinzu; fassungslos verstumme ich.

Iberisch, Keltisch. Die Trennung der beiden Sprachen voneinander ist sehr wichtig, aber einen entscheidenden Einflufs auf die "iberische Frage" hat sie nicht. Dennoch sucht V. auch auf diesem Grenzbezirk nach Waffen gegen mich. Zunächst sagt er allerdings (S. 2 I I): "si les races peuvent se mêler, les langues ne se mêlent point. Les Celtibères parlaient donc un idiome celte avec des mots et des tournures ibères, ou ibère avec des tournures celtiques. " Die letztere Behauptung, welche mit der vorhergehenden in schroffem Widerspruch steht, wird S. 235 in etwas anderer Form wiederholt; sie deckt sich mit dem was ich S. 65 (unten) ausgesprochen hatte. An der zweiten Stelle heifst es nun aber: "Il est du reste remarquable que l'escuara moderne, dont le vocabulaire contient tant de mots latins, offre si peu d'éléments celtes, s'il est vraiment apparenté à l'ancien ibère." Wie kann uns denn das wundernehmen, wenn "il est remarquable que dans aucune inscription [iberische] n'apparaissent des mots d'emprunt, latins, celtes, puniques" (S. 2 I I), was übrigens, bei unserem Verhältnis zu den Inschriften, eine recht kühne Versicherung ist? Die keltischen Lehnwörter die sich bisher im Baskischen haben ermitteln lassen, sind zwar an sich nicht allzu zahlreich, aber doch den Umständen nach nicht zu wenig; dafs so viele davon zusammen mit echtbaskischen verschwunden sind, daran sind eben die lateinischen und romanischen Lehnwörter grofsenteils schuld. Und wir kennen sogar keltische Elemente die das Baskische mit dem Iberischen gemein hat, so -ko (vgl. -aca, -aga) und tegi, -gi. Das verkennt V. nun wieder; er sagt (S. 236) dals viele von den hispanischen Ableitungen baskischen Anscheins rielmehr keltisch seien, so -ko, -iko. Wäre nun aber auch im Baskischen gar nichts Keltisches enthalten, so würde das nicht das Geringste gegen seine Zugehörigkeit zum Iberischen beweisen; im Norden konnte ja das Keltische nachdrücklicher abgewehrt worden sein. Ein merkwürdiges Verfahren schlägt V. auch S. 236 ein. Er zählt zwei Dutzend Eigennamen aus hispanischen Inschriften auf, 1 und setzt hinzu: „Bien habile

1 Die Liste ist durch einige Druckfehler verunstaltet: atecina st. ataecina, cerepaeci st. cerenaeci, bandiaeapolisegus st. -osegus, mantunaecus st. cantunaecus, crougintoudadicoe st. -igoe. In welcher Inschrift steht ongiamunaeius oder etwas ähnliches? ist es vielleicht = aegiamunniaegus? 
celui qui verra dans tout cela des radicaux basques! « Das glaube ich gern, jene Wortformen sind ja, wie V. selbst gleich darauf zugibt, wenigstens den Stämmen nach keltisch oder doch insgesamt uniberisch. Die Logik erheischte es iberische Namen vorzuführen um sie auf ihre Ähnlichkeit mit baskischem Sprachgut zu prüfen; das Vorhandensein solcher zu leugnen würde allerdings auf die einfachste Weise die niberische Frage" lösen. Es ist bekannt wie stark unter den hispanischen Personennamen diejenigen keltischen Ursprungs vorherrschen; aber sie herrschen doch eben nur vor, selbst zufolge A. Carnoy Éléments celtiques dans les noms de personnes des inscriptions d'Espagne, Louvain 1907 (aus dem Muséon $\mathrm{Bd}$. VIII), der überdies geneigt ist manche Namen für keltisch zu halten die es gewils nicht sind, z. B. Urcico (von (p) orko- „Schwein"), Elurico (von (p) etro- "Vogel ") S. 8 (s. Iber. Dekl. S. 36. 51); bei Veliagun S. 8 hat sich sogar das bask. belia (ohne Artikel bele) "Rabe" in ein keltisches Wort verwandelt. Die grolse Menge iberischer Ortsnamen wird doch auch V. nicht in Abrede stellen wollen?

Münzaufschriften. Die Lesung dieser und die Deutung der Ortsnamen sind die beiden Grundpfeiler für das Studium des Iberischen. Ich habe $\mathrm{V}$. vorgeworfen dals er das was jahrzehntelange Arbeit so vieler Numismatiker errungen hat, ohne ein Wort der Kritik beiseite schiebt, um ohne ein Wort der Begründung einen Einfall von sich bezüglich des $-s(-s)$ und des -cen u. ä. uns aufzutischen. 1 Und er wundert sich dals ich diesen Einfall nicht diskutiere (S. 230)!

Inschriften. Sie sind mir wie ihm fast ganz unverständlich; was ich für die Deklination ihnen entnehmen zu dürfen glaube, wird im folgenden Absatz berührt werden; hier beziehe ich mich nur auf Stammhaftes zu dem sich Baskisches vergleichen läfst. V. kann der Verlockung nicht widerstehen die Versuche der „Baskomanen" $z \mathrm{u}$ verbessern. Ich hatte bei der Form nersealn (-sna-) an Zusammenhang mit neska(to) "Mädchen" gedacht, welches für *ner-ska (-ska ist Deminutivendung) stünde, hatte zugleich aber auf ner(h)abe „Bursche" verwiesen. V. sagt (S. 235): nje crois plutôt qu'il convient de le rapprocher [nämlich die zuerst genannte Form] de nerhabe 'domestique, célibataire mâle', dérivé de yabe 'maître'." Diese Etymologie ist mir zwar schon der Bedeutungen

1 Die betreffenden Formen (S. 6) weisen in 7 Zeilen nicht weniger als 13 Druckfehler auf:

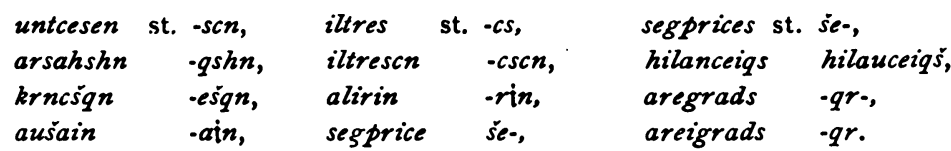

Zoitschr. f. rom. Phil. XXXII. 
wegen bedenklich, aber ich verstehe sie; wie jedoch jabe eine Ableitung von jaun "Herr" vermittelst be "unter" sein könnte ("ce serait le 'magister operum' p. ex., le commandeur des esclaves"), das verstehe ich nicht. Denn jabe, jaube unterscheidet sich im Sinne nicht wesentlich von jaun "Herr" (vgl. jabetu, jaubetu, jaundu "sich bemächtigen"), der Form nach vielleicht wie giza- von gizon ${ }$ Mensch" (-b- vor Vokal $=-u-$, wie in arabera neben arau usw.; s. Uhlenbeck Vgl. Lautl. S. 34 f.). - Von der Formel are $\vartheta g$, aredc, are $\cdot d c$ (nicht are $\cdot d e$ ), aredk steht es, auch ohne dals wir uns des baskischen Schlüssels bedienen, ziemlich fest dafs sie besagt: „hier ruht". Man hat dann angenommen dafs die beiden letzten Buchstaben dem bask. dago "er ruht" (auf Grabsteinen) entsprechen; V. hingegen sagt (S. 223): "Si l'on tient pour le basque, il vaudrait mieux lire aren duk 'tu l'as en ce lieu'." Doch äufsert er sofort inbezug auf das aren eiren Zweifel, da $h$ - oder $k$ - fehle. Mein Bedenken ist das dafs aren nicht "hier", sondern "dort" ("locatif de ar, cela") bedeuten würde $\{$ bask. (h)an. Was $d u k$ anlangt, so fragt es sich ob sich die Duzform für $d a$ "ist" in so entlegener Zeit überhaupt schon entwickelt hatte, und ferner ob sie bei einem solchen Falle anwendbar wäre, oder gar auf baskischen Gräbern angewendet wird. Gegen mein dago wendet V. ein dals "dago 'il demeure' est abusivement employé pour $d a$ 'il est', par analogie avec l'espagnol où estar remplace souvent sera". Die Sache verhält sich eher umgekehrt. In unsern Sprachen pflegt der Unterschied zwischen Zuständlichkeit und Kopula verwischt $z u$ sein, das heilst für beides nur ein Verb zu gelten: esse, sein usw. Im Romanischen der Pyrenäenhalbinsel aber tritt er scharf hervor, sodals trotz mancher ähnlichen Gebrauchsweisen des Südfranzösischen und Italienischen an den Einflufs einer vorarischen Sprache gedacht werden darf. Wenn demnach "wo ist er?" im Span. donde está? und im span. Bask. non dago? heifst, so halte ich das letztere gegenüber dem franz.-bask. non $d a ?$ gerade deshalb für das Ursprünglichere weil das erstere gegenüber dem lat. ubi est? (südfranz. ounte es?, ital. dov' d?; aber ounte esto?, dove sta [di casa]? "wo wohnt er?") etwas Sekundäres ist.

Deklination. In ihrem Mittelpunkt steht für mich der Genetiv. Ich erkenne in dem-qum, -cum, -cun welches in den lat. Inschriften so häufig als Endung von Geschlechtsnamen erscheint, den Genetiv adjektivischer Bildungen auf $-k o$; diese Endung finde ich wieder in dem -kon iberischer Inschriften und dem -qm, $-q n$ iberischer Münzen; ich stelle ihr gegenüber einerseits das $-q \xi$ der Münzen als andern Kasus (Instr.?) derselben Adjektive auf $-k o$, anderseits das -cen der Münzen als Gen. Plur., entdecke - gin als solchen auch in einer Inschrift und vergleiche erst zum Schlufs die bask. Genetivendung -en (Plur. *-ken). Man sollte meinen, V. habe alles das gar nicht oder mit der grölsten Flüchtigkeit gelesen wenn er frägt (S. 225): „Pourquoi $n$ ou $m$ est-il plutôt génitif qu'autre chose?" 
und wenn er fürchtet „que Schuchardt, songeant au basque, n'ait obéi à une sorte d'auto-suggestion". Ja, indem er fortfährt (S. 226): „ken qui est le génitif pluriel basque, est-il vraiment le correspondant des $c n, c m, q m$, om, gin etc. ibères?" und "d'autre part n'est-il pas aventureux et hardi d'assimiler cen, gin et com?" wirft er den Schein einer Verwirrung auf mich für die er ganz allein verantwortlich ist; denn ich scheide auf das Bestimmteste -ce-n, -qo-m und -om voneinander als Endungen ganz verschiedenen Wertes. Dass neroncen nicht bedeuten könne "der Narbonner" (Gen. Pl.), würde er wohl auch nicht so kurzweg behauptet haben wenn er seiner Aufmerksamkeit gewürdigt hätte was ich S. $37 \mathrm{f}$. inbezug auf jene Form sage. - Die Dativendung - $i$ erschliefse ich aus Grab- und Weihinschriften. U. a. aus IV bei Hübner: nucei $\|$ ltra $\| z u i$ (der drittletzte Buchstabe kann auch anders gelesen werden). V. gibt sie (S. 23I) mit willkürlich gesetzter Worttrennung und mit $k$ statt $c$ wieder: nuke illra $z u i$, und da kommt allerdings das letzte Wort als für einen Dativ zu kurz heraus. Meine Erklärung von crougin toudadigoe ${ }^{1}$ als "der (Gottheit) von (dem)

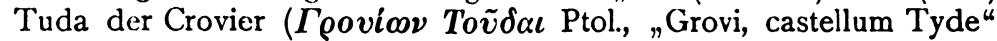
Plin.) habe ich so genau analysiert dals wenn man auch die Begründung unzureichend fände, man doch über meine Auffassung nicht im ungewissen sein könnte. Und nun sagt V. (S. 234) von dieser und entsprechenden Formen: „on n'explique pas les $e$ ou $i$ ajoutés: est-ce le signe du datif? Ce seraient alors des datifs indéfinis, mais quel en serait le sens?" Es sind allerdings Dative ohne Artikel, aber aus einer artikellosen Zeit. V. weifs dafs ich die Entstehung des Artikels aus dem dritten Demonstrativpronomen (har- „jener") vermutungsweise in etwa dieselbe Zeit wie die des romanischen Artikels setze ${ }^{2}$ und deshalb gewisse Deutungen Giacominos beanstandet habe (Ztschr. 23, 177f.). Ich weirs nicht was es nun heifsen soll, die Basken würden heute, mit dem Artikel und mit -ko statt -tiko, Tudakoari oder noch eher Tudatarrarz sagen. Wenn es bask. heifst - ich nehme das eine Beispiel Azkues - leku urrunetiko berria "die von einem fernen Orte kommende Nachricht", so wird wohl für das Iberische ein Tudatiko "von Tuda stammend“, "nach Tuda gehörig" glaublich sein. Ich gebe V. seine

1 Ich vermute dafs Touda einem kelt. Toutā im Sinne des osk. touto "Stadt" entspricht; die Stimmhaftwerdung der intervokalischen Tenuis, die in dieser kurzen Inschrift nicht weniger als dreimal vorkommt (zu dem -gin für -cen vgl. -gun für -cun) würde in dem Ortsnamen so zu sagen amtlich geworden sein.

2 V. selbst neigt sich wohl dieser Ansicht zu wenn er (S. 215 f.) meint dals in der baskischen Grammatik die artikellose Form früher eine wichtigere Rolle gespielt habe als jetzt, wie man noch sage errege (nicht erregea) "der König". Ebenso wie ich (Ztschr. 30, 7) sieht er in dem -eta- gewisser Pluralkasus artikellose Formen, in deren sonstiger Beurteilung er allerdings ganz von mir abweicht (S. 229 Anm. 2). Über das - $a$ in iberischen Ortsnamen wie Iria, Urbicua (vgl. Ztschr. 32, 80) bin ich noch nicht ins klare gekommen. 
Äufserung über Giacominos Deutungsversuche zurück: „le linguiste italien rapproche l'ibère du basque moderne contemporain .... le basque a certainement changé depuis dix-neuf ou vingt siècles" (L'Année linguistique I, $177 \mathrm{f}$.). Mit demselben Unrecht vermifst $\mathrm{V}$. in -ko-n den Artikel; ,ajouter à ce $k o$ le $n$ génitif ne signifierait rien en basque" (S. 234); nein, aber warum auch im Iberischen nichts? - In einen starken Irrtum ist V. S. 233 verfallen: „On peut remarquer aussi que Sch. dresse des listes de suffixes particuliers, les uns aux légendes monétaires, les autres aux inscriptions; il y auroit eu, dans la même langue, deux systèmes différents; p. ex. le suffixe, $c n$, cen des médailles deviendrait ein dans les inscriptions." Zunächst ist -ein, -ein ein typischer Ausgang von Genetiven (wie -scen, -cen, -tn, -qm), nicht die Genetivendung selbst, welche $-n$ (-en) lautet. Ich habe ferner gesagt dafs -ein ein Genetiv des Plurals sein könnte und dann $\mathfrak{i}$ für $c$ stehen würde wie in der Münzaufschrift salirin neben salircn, die V. selbst anführt wie um mich zu widerlegen. Umgekehrt habe ich -gin, das nur eine Lautvariante von -cen ist, inschriftlich belegt. V. schliefst diesen Absatz mit den Worten (S. 234): "Je pourrais, pour tous les suftixes pro-

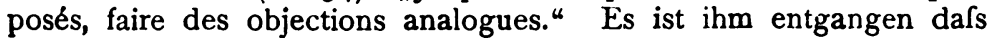
ich zwar den Instrumental auch, aber den Dativ und Aktivus nur in Inschriften vorgefunden habe. Meine Übersicht der iberischen Deklination, die ja auch von V. S. 224 wiedergegeben worden ist (was bedeutet Erit. statt meines Instr.?), enthält allerdings Doppeltes, aber ohne irgend welche Beziehung auf den Unterschied zwischen Inschriften und Münzaufschriften (Gen.: $-n$, nach $o$ oder $u$ : $-m$ usw.). - Endlich sucht V. die Unhaltbarkeit meines Kasussystems durch eine sehr eigentümliche Probe darzutun: die 21 Wörter der Inschrift von Castellón würden demselben zufolge lauter Kasus sein. Da nun aber V. sowenig wie ich ein Nominativsuffix im Iberischen annimmt, so mufs er gleich von vornherein die Möglichkeit zugeben dafs neun Formen dem Verb angehören. Und er hätte doch bedenken sollen dafs der Auslaut allein die Wortkategorie nicht erkennen lälst, wie lat. auris und audis, anni und veni, anno und amo usw. zeigen; warum soll denn einzig und allein noch sinekten dem Verb zugezählt werden dürfen? 1 Und zu allem Überflufs bemerke ich dals wenn man den Kasus die Endungen zuteilt welche V. für sie vermutet, man keine günstigere Statistik erhält. Trotz alledem kommt V. zu dem Schlufs: "l'arrangement grammatical serait tout à fait étrange. Les mêmes difficultés se présenteraient avec tout autre document" (S. 233). Hat man wohl überhaupt das Recht zu sagen: „Décidément, en tout ceci, on raisonne vraiment trop par à peu près" (S. 226), wenn man sich solcher

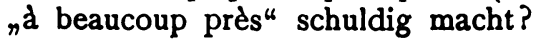

1 In dieser Inschrift würde ich nach V. (S. 230) das 18. Wort argtco in arstco verbessert haben; davon findet sich bei mir keine Spur. 
Ortsnamen. Aus ihnen hat man die erste Vorstellung vom Iberischen gewonnen und sie sind für dessen Wortstämme die einzige Quelle geblieben. Diese weisen ebenso deutlich wie die Nominalendungen der Inschriften i. w. S. auf das Baskische hin. So schlagende Übereinstimmungen wie Calagorris (im zweiten Teil) Ibarra, Ibarca (Iber. Dekl. S. 4) Iturissa, Urbicua, Biscargis (Ztschr. $32,80 \mathrm{ff}$.), als trügerisch $\mathrm{zu}$ erweisen, hat niemand sich ernstlich bemüht; auch jetzt begnügt sich V. mit dem dogmatischen Ausspruch (S. 2 IO) dafs er in den iberischen Überresten weder Wörter, noch Wurzeln, noch grammatische Formen, noch "facies général" erblicke die an das Baskische erinnerten. Aber da er des "Steckenpferdes der Baskomanen" - in Wahrheit ist es nur eines unter vielen - gedenkt, nämlich des für verschiedene Orte bezeugten Iliberri o. ä. (S. 2 I 2 f.), so mufs auch ich darauf zurückkommen. Obwohl er unmittelbar vorher gesagt hat: "il faut d'abord être sûr de la forme des mots ibères", führt er als in den iberischen Ortsnamenverzeichnissen enthalten "iliberi, illiberi, illiberri, etc." an. Ich habe gezeigt dals Illiberri ein Schreibfehler Kieperts ist und wo Iliberi steht, weifs ich nicht - die einzige wirklich, nämlich durch die Inschriften beglaubigte Form: Iliberri ist nicht aus V.s Feder gekommen. Ich sage, das ildurir der Münzen könne sachlich, aber nicht dem Laut nach dasselbe sein wie Mliberri; V. sagt: „je n'explique pas comment ildurir ou ilidurir [diese Lesung wird durch nichts empfohlen] a donné la graphie latine iliberi [so]". Kurz, an dem sicher iberischen lliberri wird mit kleinen Störsen gerüttelt, um die fast oder ganz identische Gleichung mit bask. Ili-, Iri-, Uli-, Uri-, -berri, -barri leichter umwerfen zu können. V. sagt: „en phonétique générale, $r$ a précedé $l^{\prime \prime}$. Woher weils das V.? Oder vielmehr welchen Sinn hat überhaupt dieser Satz? Ich vermute dafs V. die indo-eranische Ursprache, die das $l$ der arischen Ursprache durch $r$ ersetzt hat, mit der letztern verwechselt. Ich bringe Beispiele lateinischer Wörter deren $l$ im Bask. zu $r$ geworden ist, wie caelum \{ zeru. V. entgegnet: „Mais, je remarque que tous les exemples sont en $e$ ou $u$ [und soro neben solo, dem sich andere Fälle wie borondate anschliefsen?] et pas en $i^{\text {u, }}$, übersieht jedoch dabei oritz neben olitz, und anderes von mir nicht erwähntes, auch echt baskisches, so bizk. estari für das in allen übrigen Mdd. erhaltene estali. $\mathrm{Er}$ fährt fort: „or, $i$ a certainement plus d'affinités avec $l$ qu'avec $r$ "; das hätte er vom physiologischen Standpunkt erweisen und mit Beispielen aus der Lautgeschichte erläutern müssen. Alles das zeigt dafs V. seinen Widerspruch nicht aufgeben will und doch nicht begründen kann.

Ilit-. S. 226 hält mir V., nach der Bekämpfung meines iberischen Kasussystems, „un autre exemple de raisonnement défectueux" vor. Ich vergleiche iberische Zusammensetzungen die in doppelter Form bezeugt sind, wie Iliturgi = Ilurgi mit baskischen 
wie begitarte von begi und arte usw. Wenn ich auf solche andern Erklärungsmöglichkeiten, wie sie $\mathrm{V}$. anbietet, keine Rücksicht nehme, so sehe ich das nicht als Mangel an. Denn es handelt sich bei ihnen um Lautveränderungen die nicht $z \mathfrak{u}$ erweisen, auch durch keine Analogie wirklich zu stützen sind, vor allem um den Übergang eines intervokalischen $k \quad z u l$. Man hat diesen in baitaiz "weil du bist" und entsprechenden Fällen finden wollen, aber die Analyse: $b a i+k a i z^{*}$ \{ haiz ist nicht richtig; das Präfix wird nicht von bai-, sondern von bait- gebildet (s. Museum X, 403, und dies ist auch Azkues Auffassung). Dagegen lärst sich auch baihincen bei Leiçarraga (s. Einl. zu L. S. XL) neben baitinģan bei Dechepare "weil du warst" nicht ins Treffen führen; jenes beruht auf Analogiewirkung. In baikare \{ bai + gare "weil wir sind" könnte an sich zwar die Tenuis durch den vorhergehenden Diphthongen hervorgerufen worden sein; aber dann mülsten wir fragen warum ist hieraus nicht baitare* geworden wenn baitaiz, baitinçan wirklich für *baikaiz, *baikinçan stehen. Legen wir aber bait- zugrunde, so erklärt dies sowohl baitaiz, wie baikare (für *bait-gare), und wir gewinnen eine genaue Parallele zu begitarte und bepuru "Augenbraue" (für *begitburu "Augen-haupt"), bekaitz "Neid" (für *begit-gaitz "Augeschlimmes"). Allerdings gibt es ein paar Zusammensetzungen mit su "Feuer" in denen -k- und -t- miteinander wechseln: sukopil, sutopil "Aschkuchen", sukalde, sutalde (eig. „Feuerseite") „Küche“, "Herd"; aber hier muls das $k$ zum ersten Worte gehören (vgl. sukatu "brennen"), um so mehr da opil \} lat. ofella ist, und alde "Seite" möglicherweise / rom. falda, halda "untere Seite", "Ausläufer eines Berges".

Ich habe Vinson nicht überzeugt, und er mich nicht. Nun meint er zwar dals solche Erörterungen nicht ohne Nutzen seien; das gilt aber doch nur dann wenn sich wirklich zwei Hypothesen gegenüberstehen oder wenn mit den Verneinungen und Bezweiflungen irgend welche positiven Darlegungen verknüpft sind. Ist auf den gegebenen Tatsachen ein unsicheres Gebäude errichtet worden, so reifse man es nur ein um ein festeres an seine Stelle zu setzen. Sind die Basken nicht der kleine Úberrest eines grofsen Volkes, so beleuchte man die Umstände unter deren Gunst das Baskische wesentlich in den gleichen Grenzen und zwar keineswegs von der Nalur sehr geschützten sich zwei Jahrtausende gegen Römer und Romanen zu halten vermochte; beweisen Ortsnamen wie Iliberri, Biscargis usw. nichts für die Verwandtschaft zwischen Iberisch und Baskisch, so zeige man wie leicht und wie häufig der Zufall solche Übereinstimmungen herbeiführen kann; bieten die Denkmäler jener alten Sprache im ganzen zu wenig Anklänge an diese neue, so setze man das durch Gegenüberstellungen anderer Verhältnisse in helles Licht, z. B. desjenigen zwischen den libyschen Inschriften und Ortsnamen und dem Berberischen; sind 
die iberischen Endungen -cum, -cun u. ä. falsch als Genetive gedeutet, so bringe man eine bessere Erklärung von ihnen vor usw. usw. V. schliefst mit dem baskischen Sprichwort: Wer mit "vielleicht" redete, täuschte sich nicht. Ich setze hinzu: aber traf auch nicht ins Schwarze. 1

1 Ich erlaube mir hier einen sinnstörenden Druckfehler Iber. Dekl. S. 67 Z. $17 \mathrm{zu}$ verbessern: "dissimilierende" in "mouillierende".

Nachschrift. Zu S. 351 Anm. Z. 18 von unten.

In dem mir eben zugekommenen 2. Heft der Rev. intern. des Et. Basques von I 908 lese ich S. 255 in einer kurzen Anzeige Vinsons von Azkues Wörterbuch: "aztigar ou gaztigar n'est-il pas plutôt 'tilleul' que 'érable'?" Ich möchte die Gegenfrage an Vinson richten - die er wohl aus der Erfahrung seiner Jugend leicht beantworten kann - ob denn die Linde nicht ein verhältnismälsig seltener Baum bei den Basken ist. Daher würde sich die Unsicherheit ihrer Benennung erklären (man sehe besonders ezki bei Azkue; Lacoizqueta gibt diese entschieden als Namen der Linde an). In einem eben zu St.-Jean-de-Luz erschienenen Büchlein von Guilbeau: Quelques extraits du vocabulaire basque enthält das Verzeichnis der Bäume S. 19-2I die Linde gar nicht; der Ahorn aber heilst auch hier astigar, gastigar.

H. Schuchardt. 